\title{
臨床報告
}

\section{A Novel Bite-raising Procedure for Complete Dentures}

\author{
Noboru KitagaWA and Yuji SATO \\ Department of Geriatric Dentistry, Showa University School of Dentistry
}

\begin{abstract}
Complete dentures with decreased vertical dimension should be bite-raised as treatment dentures before new complete denture treatment. The aim of this study is to present a new procedure to increase the occlusal vertical dimension of complete dentures. In this paper, a novel full-arch, bite-raising procedure for complete dentures with a silicone putty core is described to have better appearance. The procedure includes mixing a putty-type siliconeimpression material and making an impression of the occlusal and polished surfaces with a stock tray; removing the dentures and abrading the surface of the artificial teeth; pouring the mixed autopolymerizing resin in the artificial tooth depression of the impression; repositioning the putty-type silicone-impression material core on the denture and applying pressure to it. This technique is simple at chair side. Laboratory procedures require an extra appointment. This technique can decrease the number of appointments. This simple procedure is effective for complete dentures with decreased occlusal vertical dimension. This technique is easy, fast, accurate, inexpensive and readily available to clinicians.
\end{abstract}

Key words: increased occlusal vertical dimension, clinical procedure, complete denture.

To maintain a harmonious stomatognathic system, the dentist must establish an appropriate occlusal vertical dimension for complete dentures. ${ }^{1 \sim 4)}$ Complete dentures with decreased occlusal vertical dimension should have occlusal reconstruction as treatment dentures before new denture treatment is completed. ${ }^{5)}$ This is because decreased occlusal vertical dimension causes masticatory dysfunction, esthetic dysfunction, disorders of articulation and temporomandibular disorders. ${ }^{6)}$ Intraoral procedures with an autopolymerizing resin ${ }^{7)}$ (autopolymerizing resin added to the occlusal surface of artificial molar teeth) are timeconsuming and result in ill-appearance. Laboratory procedures require an extra appointment. In this paper, a novel full-arch occlusal vertical dimension increase procedure for complete dentures using a putty-type siliconeimpression material core is described to have better appearance. Additionally, this technique is low in cost compared to making new dentures.

\section{Materials and Methods}

Using a clean tissue, polish the surface of the old denture. Mix the putty-type siliconeimpression material (EXAFINE; GC Corp., Tokyo, Japan), and make an impression of the occlusal and polished surfaces using a stock tray (DENTULOUS IMPRESSION TRAY; Dentsply-Sankin Corp., Tokyo, Japan). Mark cross lines at the impression margin in the anterior and posterior portions with a sharp indelible felt-tip pen (Fig. 1). Remove the dentures and abrade the surface of the artificial teeth using airborne particulates or an abrasive point. Apply the autopolymerizing resin (UNIFAST; GC Corp., Tokyo, Japan) monomer (by adhesive) to the surface of the artificial teeth. Mix the autopolymerizing resin in a low powder/ monomer ratio. Pour the mixed resin in the artificial tooth depression of the impression (Fig. 2). Reposition the puttytype silicone-impression material core on the

(Received February 29, 2008; Accepted for publication April 23, 2008) 


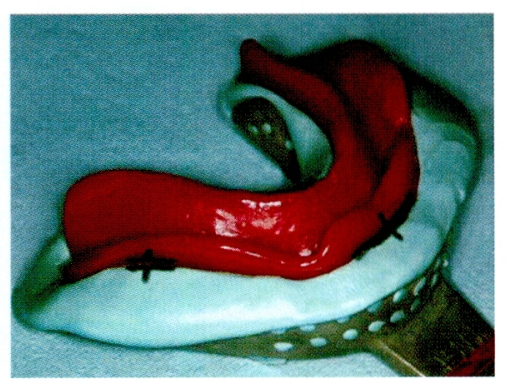

Fig. 1 Mark cross lines at the impression margin in the anterior and posterior portions with a sharp indelible felt-tip pen.

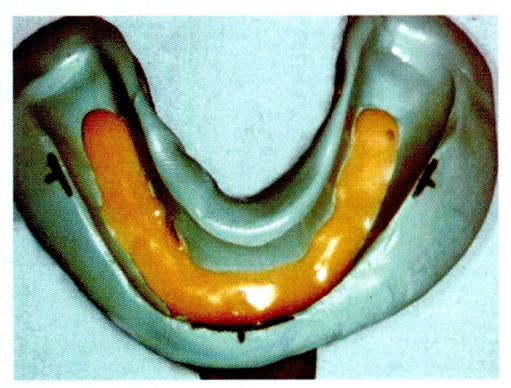

Fig. 2 Pour mixed resin in the artificial tooth depression of the impression.

dentures and apply pressure until the gap between the anterior line and putty border is at the planned height and the gap between the posterior line and putty border is half of that height (Fig. 3). Remove the denture when the resin takes on a gum-like property. Seat the dentures into the mouth and instruct the patient to close their mouth with moderate pressure. Remove the denture from the mouth and remove the excess acrylic resin on the polished surface with sharp instruments. Examine and adjust the occlusion using articulating paper. Polish the surface of the denture in a conventional manner (Fig. 4).

\section{Results}

This technique uses a full-arch bite-raising procedure for complete dentures. Not only artificial molar teeth, but also artificial anterior teeth were bite-raised.

\section{Discussion}

Complete dentures with decreased occlusal vertical dimension should usually be subjected



Fig. 3 Reposition the putty-type silicone-impression material core on the denture. The gap between the anterior line and putty border is at the planned height, and the gap between the posterior line and putty border is half of that height.

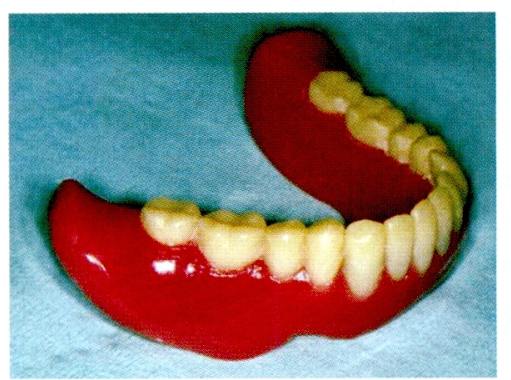

Fig. 4 Occlusal vertical dimension of the complete denture is increased.

to bite raising. There are two methods of bite raising; namely, direct and indirect. The indirect method is very reliable but requires laboratory work. This new method (direct method) does not require such work.

Intraoral procedures (conventional direct method) with an autopolymerizing resin are difficult. Especially difficult is reconstructing the occlusal surface of artificial molar teeth. However, this method is simple and easy. Furthermore, it is distinctive in that autopolymerizing resin is added not only to the artificial molar teeth, but also to the fullarch at chair side.

The advantages of the method are reductions in laboratory procedure time and cost, and the autopolymerizing resin is inexpensive. Furthermore, there are some situations where this method is preferable; especially in cases where complete dentures are not kept at the laboratory. 


\section{References}

1) Hammond RJ, Beder OE: Increased vertical dimension and speech articulation errors. J Prosthet Dent, 52: 401-406, 1984

2) Babu CL, Sing $S$, Rao SN : Determination of vertical dimension of rest: A comparative study. J Prosthet Dent, 58: 238-245, 1987

3) Mohindra NK, Bolman JS: The effect of increasing vertical dimension of occlusion on facial aesthetics. Br Dent J, 192: 164-168, 2002

4) Mayas KA : Reestablishing occlusal vertical dimension using a diagnostic treatment prosthesis in the edentulous patient: A clini- cal report. J Prosthodont, 12: 30-36, 2003

5) Kurihara M, Takahashi M, Ohkawa S, Amano $\mathrm{H}$ : Reestablishing occlusal vertical dimension by use of a diagnostic treatment denture in the edentulous patient: A case report. Meikai Univ Dent J, 33: 261-265, 2004 (in Japanese)

6) Yoshida Y : Effect of higher vertical dimension of full denture. 歯科審美, 13: 134-136, 2001 (in Japanese)

7) Hobkirk JA : A Clinical Atlas of Complete Dentures. London, 1985, Wolfe Medical Publications Ltd. pp 73 